\title{
MULTILINGUAL DATA MANAGEMENT IN DATABASE ENVIRONMENT
}

\author{
Abu Sayed Md. Latiful Hoque ${ }^{1}$ and Mohammad Shamsul Arefin ${ }^{2}$ \\ ${ }^{1}$ Department of Computer Science and Engineering, \\ Bangladesh University of Engineering and Technology (BUET), Dhaka-1000, Bangladesh. \\ Email: asmlatifulhoque@cse.buet.ac.bd \\ ${ }^{2}$ Department of Computer Science and Engineering, \\ Chittagong University of Engineering and Technology (CUET), Chittagong-4349, Bangladesh. \\ Email: sarefin_406@yahoo.com
}

\begin{abstract}
Global E-Commerce and E-Governance programs have brought into sharp focus for the need of database systems to store and manipulate data efficiently in a suite of multiple languages. While existing database systems provide some means of storing and querying multilingual data, they suffer from redundancy proportional to the number of language support. In this paper, we propose a system for multilingual data management in distributed environment that stores data in information theoretic way in encoded form with minimum redundancy. Query operation can be performed from the encoded data only and the result is obtained by decompressing it using the corresponding language dictionaries for text data or without dictionary for other data. The system has been evaluated by both syntactic data and real data obtained from a real life schema. We have compared the performance of our system with existing systems. Our system outperformed the existing systems in terms of both space and time.
\end{abstract}

Keywords: Multilingual Data Management, database environment, data dictionary, lexeme, query

\subsection{INTRODUCTION}

Efficient storage and query processing of data spanning multiple natural languages are of crucial importance in today's globalized world [1, 2, 3]. As Internet has become a primary medium for information access and commerce, multilingual data management $[4,5]$ in database environment can be treated as a vital issue for the availability of information in the native language of the Internet users. Survey results indicate that the demographics of the Internet are steadily becoming multilingual. The non-native English speaking users of the Internet has grown from about half in mid-90's, to about two-thirds now and it is assumed that the majority of the Internet information will be multilingual by 2010 [6]. It has been found that a user is likely to stay twice as long at a site and four-times more likely to buy a product or consume a service, if the information is presented in their native language [7]. Hence, it is important that the information systems support efficient handling of multilingual data.

Research results [8] show that significant performance degradation occurs when handling multilingual data using current database systems. An efficient Multilingual Data Management System (MDMS) is necessary to overcome the limitations of multilingual data handling capability of the existing database systems and for better searching and browsing capabilities in different languages, accessing information stored in different languages, accelerating globalization of businesses and implementing e-Commerce and e-Governance. 
We have to consider generally three main considerations for MDMS. Firstly, there should be a technique by which the data will be represented in a language-independent way. Secondly, an efficient translator is needed for performing translation among languages. Thirdly, an efficient mechanism is required to perform different types of multilingual operations in a distributed database environment [9]. These are the crucial issues in multilingual data management.

This paper presents a system to store multilingual data in a language independent way such that database evolution is easier. Queries can be performed using a translator-based approach. In this approach when information in a specific language is provided, the translator will generate its corresponding information in the target language. Schema evolution which is difficult in the existing systems, is simple and easier in this system to maintain database consistency. Query performance is also significantly faster.

\subsection{LITERATURE REVIEW}

While a rich body of literature on multilingual information processing exists in the Natural Language Processing [10] and Information Retrieval [11, 12] communities, there is comparatively very little in the database context. In database literature, the multilingual data management issues may be classified as solutions for specific languages, data integration solutions or proprietary solutions.

A database system for handling Arabic data is presented in [13]. This work presents specific issues and solutions for storing, indexing, querying and presenting Arabic language data, in an object oriented way. A database system has been presented for storing and query processing ideographic Chinese, Japanese and Korean (CJK) data in [14], where the authors primarily focus on the definition of resources needed for handling ideographic scripts in database systems. Though both these papers address issues specific to the languages concerned (Arabic and CJK languages respectively), neither of them propose solutions for multilingual data management for interchange of information among users of different languages.

The Federated Multilingual Database system (FEMUS) [15] can integrate data from different data models and the associated query languages. But the limitation of this system is that it does not address issues in integrating data from different natural languages. A multilingual queryprocessing framework for sharing lexical resources is discussed in [16]. The main focus of this work is on improving the efficiency of administration of multilingual resources in database environment. But it does not provide any guideline for multilingual query processing.

The Look-Alike-Sound-Alike [17] and EROS [18] are two application specific multilingual data management systems. These systems support multilingual data for specific domain but do not address general-purpose multilingual data management issues. The commercial database systems $[19,20,21]$ provided multilingual support to some extent but not the full functionalities of MDMS. These commercial database systems support Unicode 3.01 standard for storing multilingual data. But main limitation of these systems is that they do not provide any mechanism of querying over multiple languages.

\subsection{MULTILINGUAL DATA MANAGEMENT: SYSTEM DESIGN AND ARCHITECTURE}


The system architecture for the multilingual data management comprises two main modules: management module and storage module. The management module is responsible for performing inter-language mapping and querying. It consists of two sub modules: query execution and translation. The storage module manages the storage of the encoded database and data dictionaries. The overall system architecture is shown in Fig.1. The single directional arrows represent the direction of next sub-module to be executed in a module whereas bidirectional arrows represent the relationship among the sub-modules for processing. The relationships among the sub-modules are also shown in Fig.1.

\subsection{Management Module}

Management module consists of two sub-modules: query execution and translation.

\subsubsection{Query Execution}

The query execution module consists of a group of sub-modules that are related to process a query. These sub-modules are -query manager sub-module, query input / response sub-module, parse query sub-module, search dictionaries sub-module, dictionary-to-DB mapping sub-module, and DB-to-dictionary mapping sub-module as shown in Fig.1. Query manager sub-module performs the task of retrieving the query results to the clients.

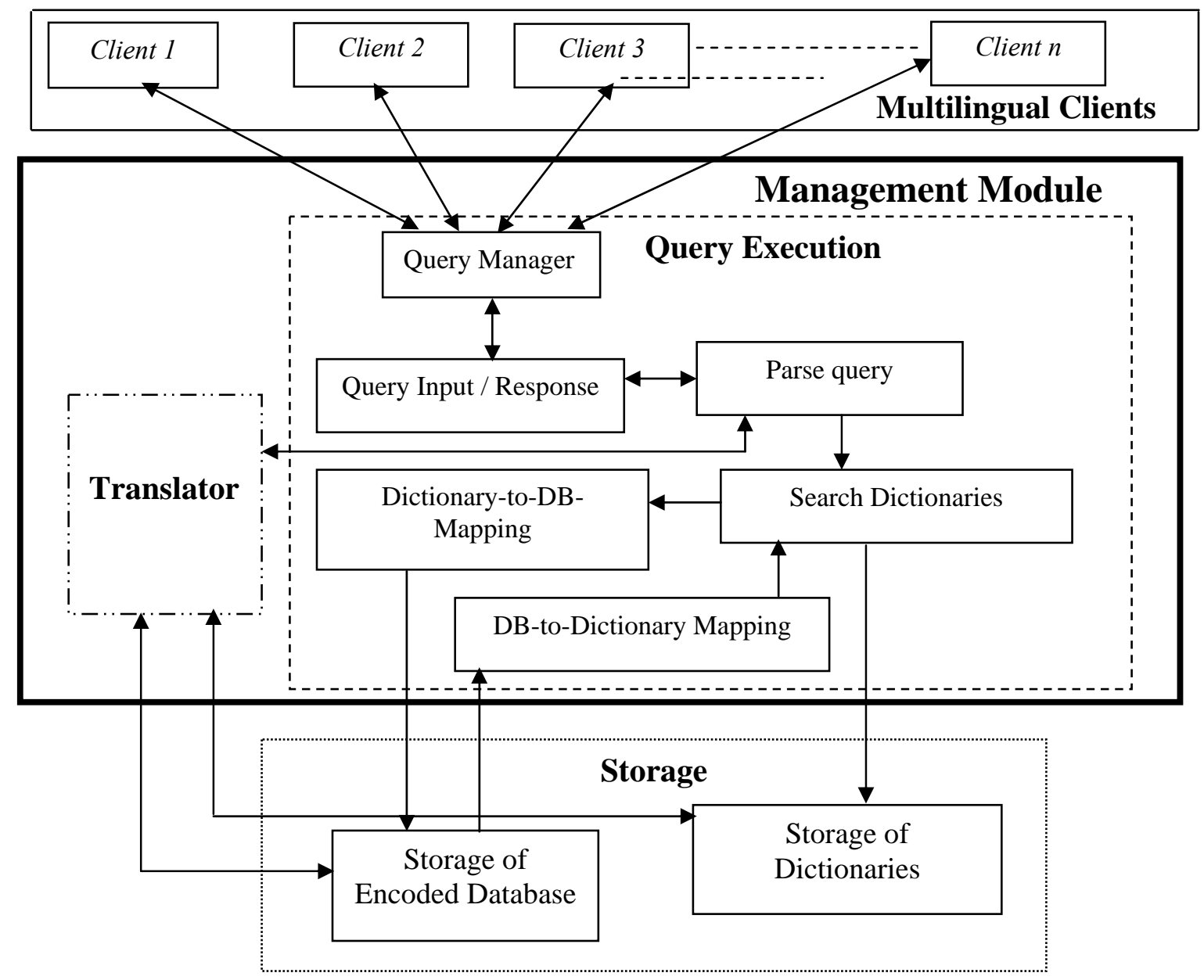

Fig.1: System architecture for multilingual data management 
Query input / response sub-module takes the query expression from the query manager and returns the query result to the query manager. Parse query sub-module identifies the important information in the query i.e. for which purpose query is generated. Appropriate dictionaries can be selected, when necessary using search dictionaries sub-module. Dictionary-to-DB-mapping sub-module performs the tasks of linking between data dictionaries and database so that retrieving of information from the database can be done easily. DB-to-dictionary-mapping submodule is used to perform reverse mapping. This sub-module helps to get the appropriate information for the client.

\subsubsection{Translation}

In multilingual translation, a record provided in specific language is translated in the target language with the help of a translator. Fig. 2 shows the multilingual translation procedure.

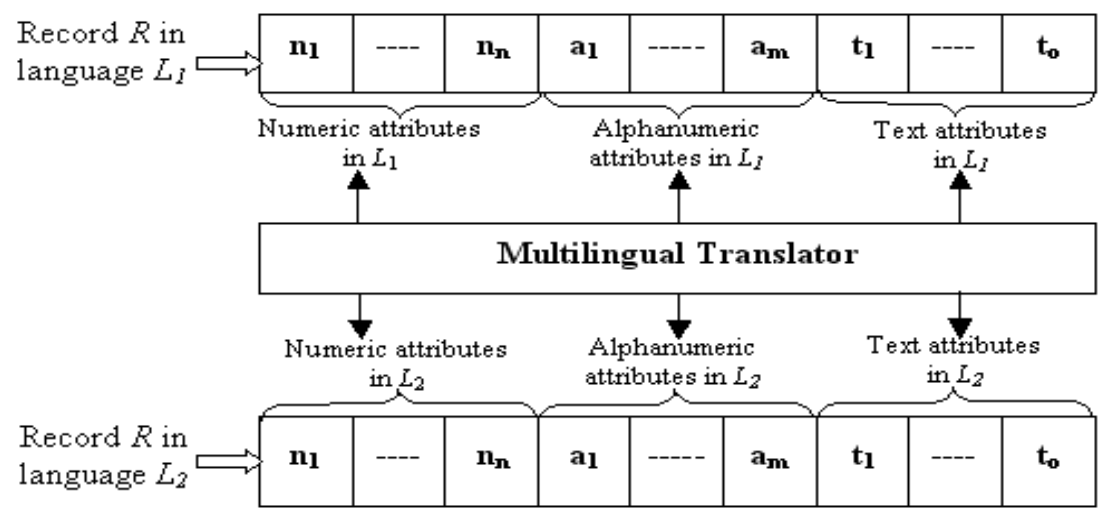

Fig. 2: Multilingual translation procedure

From the Fig. 2, it is observed that $R$ is a record in language $L_{l}$, which contains $n$ numeric attributes $n_{1,--,}, n_{n}, a$ alphanumeric attributes $a_{1},--, a_{m}$ and $t$ text attributes $t_{1,--}, t_{o}$. For translating the record $R$ in language $L_{2}$ we need to encode $R$ with the help of a translator. The translation of numeric and alphanumeric and data can be done by providing character-by-character mapping among languages while the translation of text data requires the involvement of data dictionaries. Considering the above factors the algorithm for multilingual translation is as shown below.

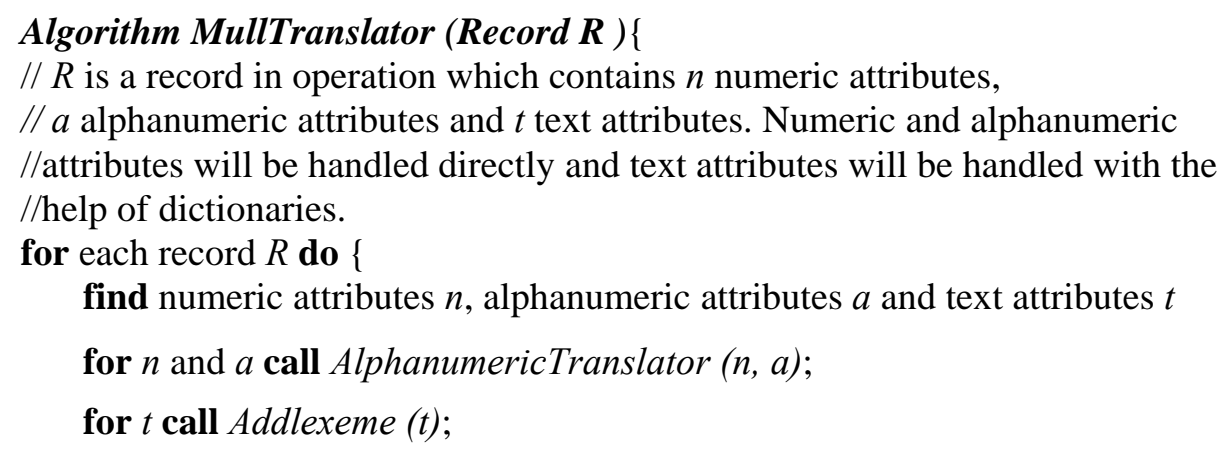

From above algorithm, it is observed that Mulltranslator algorithm takes a record $R$ as its argument and calls AlphanumericTranslator for numeric and alphanumeric attributes and Addlexeme for text attributes. AlphanumericTranslator takes numeric attribute $n$ and 
alphanumeric attribute $a$ of record $R$ as its argument and performs direct mapping with the database. The algorithm inserts numeric data in binary format and alphanumeric data in ASCII format. The algorithm for translating numeric and alphanumeric data is given below.

\author{
Algorithm AlphanumericTranslator (n, a ) \{ \\ // This algorithm takes numeric attributes $n$ and alphanumeric attributes $a$ of a \\ $/ /$ record $R$ as an argument and perform direct mapping with the database. \\ for each character $c$ in each value of $n$ or in each value of $a$ in $R$ do \\ Search appropriate character wise mapping between database storage \\ language and desired language.
}

Addlexeme algorithm takes text attributes $t$ of the record $R$ as its argument and performs translation among languages with the help of domain dictionaries. The algorithm first searches the corresponding domain dictionary for the existence of the text item in the dictionary. If the item exists in the dictionary the Addlexeme algorithm does not insert the item in the dictionary. Otherwise the algorithm inserts the item in the corresponding dictionary with its translated values in other languages.

So, there is only one entry of a specific text item in the domain dictionary. The Addlexeme, algorithm for addition of lexeme is given below.

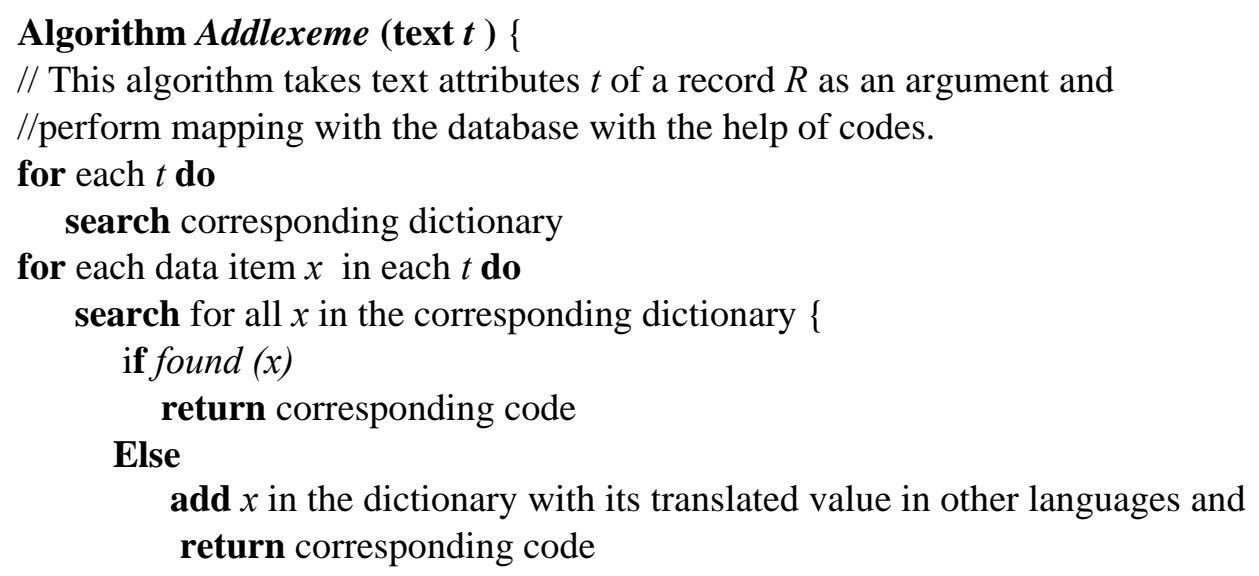

\title{
3.2 Storage
}

Data stored in the database-tables are generally text, numeric or alphanumeric. In practice most of the data is numeric and alphanumeric. Consider the relation student with attributes studentid, name, status, age and city. As an example, consider the storage of student relation in English and the native language Bangla. Table 1 and Table 2 show these two relations.

Table 1: Student_english: Storage of student records in English

\begin{tabular}{|c|c|c|c|c|}
\hline studentid & Name & status & age & City \\
\hline 0304001 & Abdullah & Married & 32 & Dhaka \\
\hline 0405006 & Abdur Rahman & Unmarried & 28 & Lakshmipur \\
\hline 0605002 & Mushfuk & Married & 30 & Dhaka \\
\hline
\end{tabular}


Table 2: Student_bangla: Storage of student records in Bangla

\begin{tabular}{|c|c|c|c|c|}
\hline studentid & name & status & age & city \\
\hline 000800$\rfloor$ & মারদুন্সুহ & बिजारिত & $\infty$ & जका \\
\hline 08 0600৬ & ঘানদূর রহম্মান & ष्্বিনাহিত & ২৮ & ন ম্মীপূর \\
\hline 0৬ం 002 & মूर्मकिष्্ & बিন্যিত & 90 & जका \\
\hline
\end{tabular}

Conventional database systems store relations for each language. So data redundancy is proportional to the number of language support. In the proposed system, a single encoded table is used to store a multilingual table irrespective of the number of languages support. Table 3 is the encoded representation of the tables (Table 1 and Table 2) in English and Bangla.

Table 3: Encoded database storing multilingual data

\begin{tabular}{|c|c|c|c|c|}
\hline studentid & name & status & age & city \\
\hline 0304001 & $1(01)$ & $1(0)$ & 32 & $1(01)$ \\
\hline 0405006 & $2(10)$ & $2(1)$ & 28 & $2(10)$ \\
\hline 0605002 & $3(11)$ & $1(0)$ & 30 & $1(01)$ \\
\hline
\end{tabular}

To create the encoded representation, we have three considerations: all numeric fields are represented directly in binary format; all alphanumeric fields are represented in character data and text fields are represented in dictionary encoding method. In Table 1 and Table 2, age is a numeric field that has the binary representation with minimum number of bits in the encoded table (Table 3). StudentID is an alphanumeric field that has been represented as ASCII character data. The other fields e.g. name, status and city are text fields and encoded using the corresponding domain dictionaries as shown in Table 4, 5 and 6.

Table 4: Dictionary for nome attribute

Table 5: Dictionary for stotus attribute

\begin{tabular}{|c|c|c|c|c|}
\hline Hame & english & baugh & $\ldots$ & --- \\
\hline 1 & Abchall at & घानासम्बाइ & -- & -- \\
\hline 2 & $\begin{array}{c}\text { Abdutu } \\
\text { Fattli ani }\end{array}$ & प्यानमाप्र ब्रद्यान & -- & -- \\
\hline 3 & Iulusthitik & यूर्निम्न्न & -- & -- \\
\hline
\end{tabular}

\begin{tabular}{|c|c|c|c|c|}
\hline status & englich & b angla & $\ldots$ & -- \\
\hline 1 & Martied & बिन्नास्ति & -- & -- \\
\hline 2 & Untrlatried & जनिनास्ति & -- & -- \\
\hline
\end{tabular}

Table 6: Dictionary for city attribute

\begin{tabular}{|c|c|c|c|c|}
\hline city & english & bangla & $\ldots \ldots$ & -- \\
\hline 1 & Dhaka & एका & - & $\overline{--}$ \\
\hline 2 & Lakshmmipur & 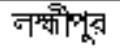 & -- & -- \\
\hline 3 & Chittagotig & होंसान & -- & -- \\
\hline
\end{tabular}

The dictionaries are created by storing only the unique values of the corresponding domain. The property of the dictionary is that if any lexeme is stored in the dictionary, it returns a unique code and vice versa i.e. code $\leftarrow$ encode (lexeme) and lexeme $\leftarrow$ decode (code). Table 4 shows the dictionary of name attribute with language 1 as English and language 2 as Bangla. There are three name instances in English and the corresponding values in Bangla in the following column. 
So, in the name field of the encoded table code 1 will represent Abdullah or प्यानमाश्र . Similarly, Abdur Rahman or घानमुत्र ज्ञा्यान and Mushfik or मून्नकिज्न will be represented using code 2 and 3 respectively in the encoded table (Table 3). Similarly, Table 5 and Table 6 show the dictionaries for status and city attributes respectively. The code corresponding to a data item stored in different languages (here in English and Bangla) will represent that data in the encoded table. So, the storage in the encoded table is independent of the number of language support.

\subsection{Operations in Multilingual Database}

Different operations such as multilingual query, insertion, deletion and update can be performed in the developed system efficiently. The following subsections describe the procedures for different multilingual operations.

\subsubsection{Analysis of Multilingual Query Procedure}

Multilingual query should be executed efficiently so that correct tuples are retrieved from the database. The algorithm for multilingual query processing is given below.

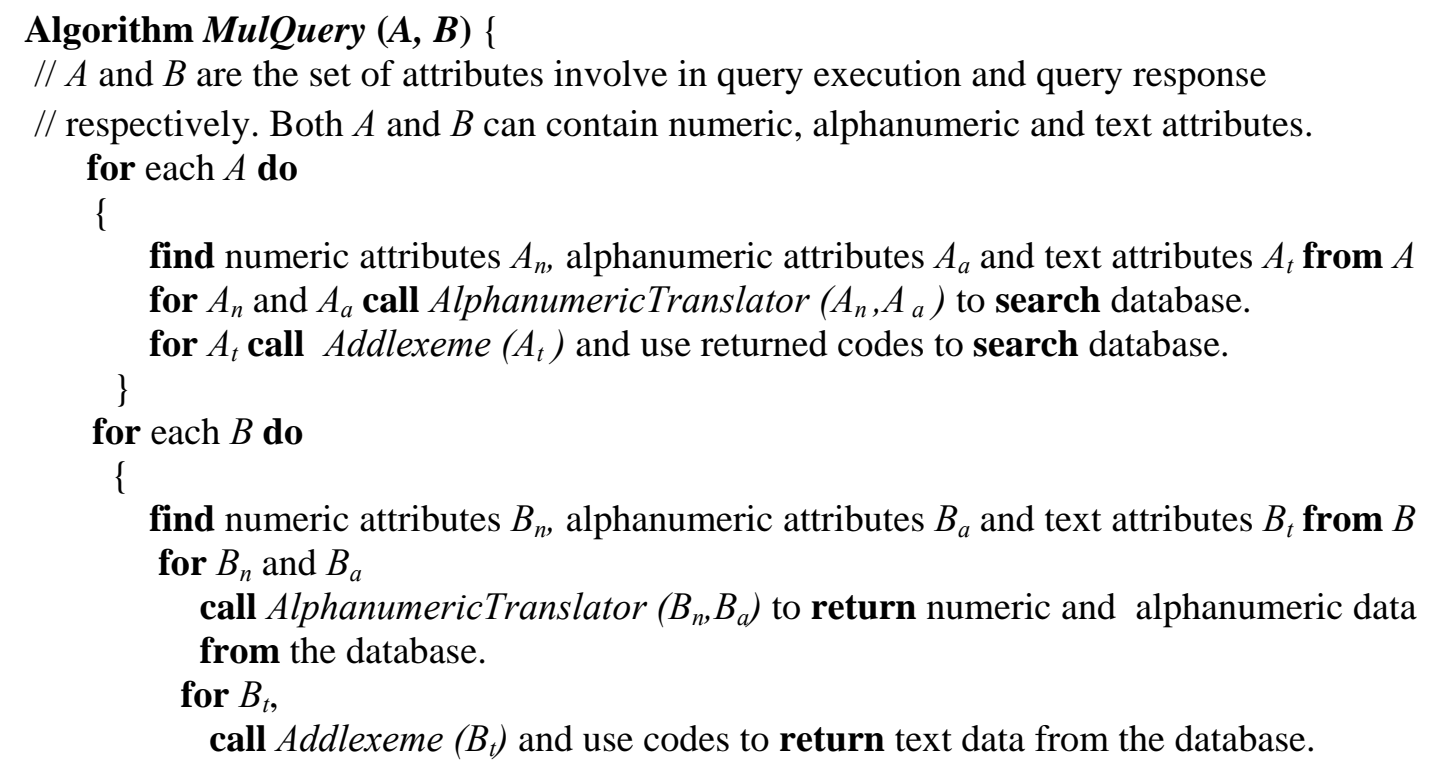

Consider the information of some students has been stored in an information theoretic approach in the multilingual database. Student record includes student ID, name, status, age and city. Name, status and city of students have been stored using dictionary encoding method and student $I D$ and age have been stored directly in the encoded DB. Now for retrieving students' records living in Dhaka city in English by a client the following is the query to be executed.

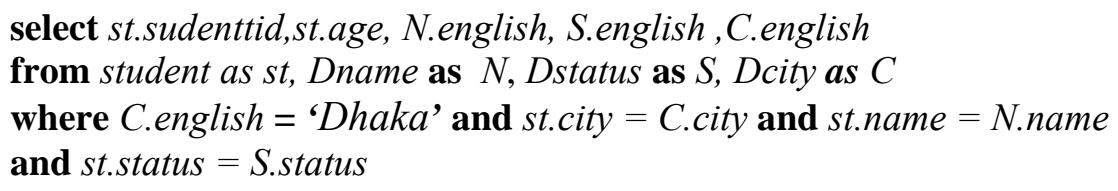

In the above query, Dname, Dstatus and Dcity are the domain dictionaries for name, status and 
city and student is the name of the encoded table. For executing the above query, algorithm MulQuery, first searches the dictionary Dcity for city attribute by calling Addlexeme algorithm. Addlexeme algorithm returns the code corresponding to Dhaka. This code will be checked in the city field of the encoded table student. Then all the matching records corresponding to the code for Dhaka will be retrieved from the database. After retrieving the records from the database, the text data i.e. name, status and city will be returned using DB-to-dictionary mapping and the numeric and alphanumeric data studentid and age will be returned directly to the client using AlphanumericTranslator in English.

\subsubsection{Analysis of Multilingual Insertion Procedure}

In multilingual insertion, care has to be taken for handling the dictionaries properly. Otherwise, data redundancy and inconsistency in the dictionary can be occurred. Also mapping among data dictionaries and database must be handled carefully for removing difficulties in accessing data from the encoded database. The algorithm for multilingual insertion is given below.

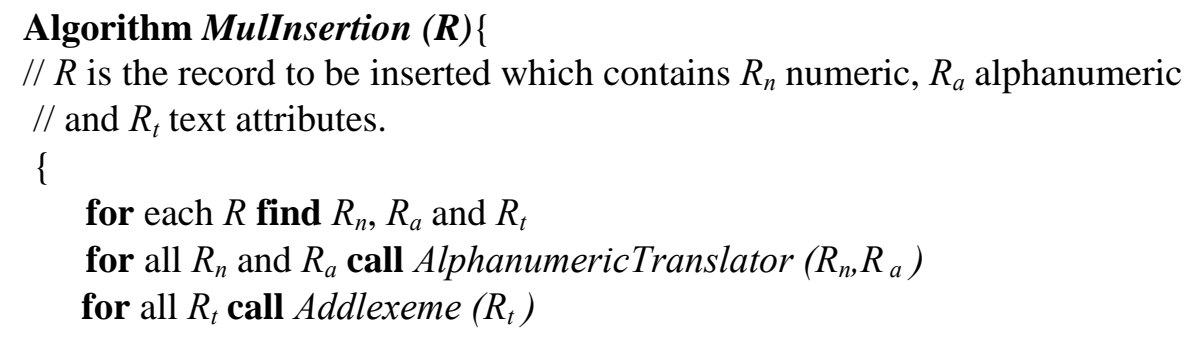

Now, consider that a client wants to insert the record shown in Table 7 in English.

Table 7: Record to be inserted

\begin{tabular}{|l|l|l|l|l|}
\hline StudentID & Name & Status & Age & City \\
\hline 0706005 & Rahim & Unmarried & 35 & Lakshmipur \\
\hline
\end{tabular}

For inserting the record shown in Table 7, the client has to execute the following SQL statements.

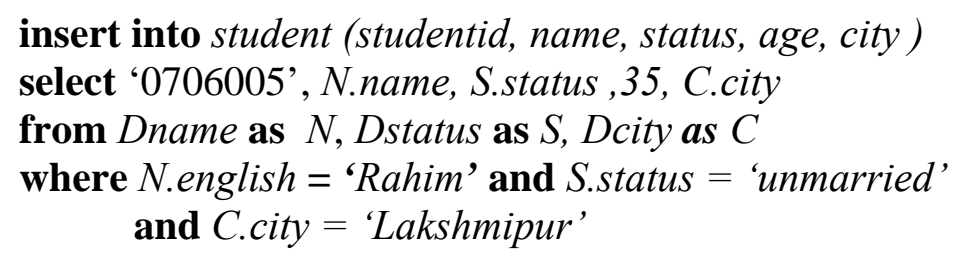

For inserting the record shown in Table 7, MulInsertion algorithm first identifies studentid as alphanumeric attribute, age as numeric attribute, name, status and city as text attributes. As studentid is an alphanumeric value and age is a numeric value they will be directly stored in the database with the help of AlphanumericTranslator. As name, status and city are text attributes the dictionaries Dname, Dstatus and Dcity corresponding to name, status and city attributes will be searched first. As dictionary Dname does not contain the name Rahim, Rahim will be inserted in the dictionary with its translated value in other languages. A code (here code 5) will be generated at the time of insertion, which will be used to represent Rahim in the database. But 
dictionaries Dstatus and Dcity contain the value unmarried and Lakshmipur respectively. So, status and city information will not be inserted in the dictionary. We just pick the codes corresponding to status and city i.e. code 2 and 2 respectively from dictionaries Dstatus and Dcity respectively. So, the record will be represented in the encoded table as shown in Table 8.

Table 8: Record in the encoded table

\begin{tabular}{|l|l|l|l|l|}
\hline StudentId & Name & Status & Age & City \\
\hline 0706005 & 5 & 2 & 35 & 2 \\
\hline
\end{tabular}

\subsubsection{Analysis of Multilingual Deletion Procedure}

To delete records from the database we will have to search the records first. The algorithm shown below performs the task of multilingual deletion.

Algorithm MulDeletion $(R)$ \{

//Delete record $R$, which contains numeric, alphanumeric and text attributes.

Call MulQuery $(A, B)$ to search for $R$

check the constraints applied and perform deletion from the database not from the dictionary.

From the above algorithm, it has been observed that deletion will be performed from the encoded database, not from the dictionary. This is because dictionary information might be needed in future for different types of multilingual operations.

\subsubsection{Analysis of Multilingual Update Procedure}

Multilingual update procedure involves querying the records. After retrieving the records update operation will be performed. The algorithm for multilingual update is given below.

Algorithm MulUpdate (R) \{

$/ / R$ is the record to be updated which contains numeric, alphanumeric

$/ /$ and text attributes.

for each $R$, call MulQuery $(A, B)$ for retrieving $R$

if the value to be updated is text then

call Addlexeme $(t)$

if the value to be updated is numeric and alphanumeric then

call AlphanumericTranslator ( $n, a)$

Multilingual update operation is more time consuming than other operations. The MulUpdate algorithm first checks the existence of the text data in the corresponding domain dictionaries with which replacement will be made. This is because if the data in the dictionary further insertion of this data in the dictionary will create data duplication. But if it is not in the dictionary we have to insert it in the dictionary with its translated value and code. This code will replace the corresponding code in the compressed database. As in translator-based approach numeric and alphanumeric data is directly inserted in the database, we will replace the numeric and alphanumeric value directly without the involvement of dictionaries. 


\subsubsection{Use of Two-Phase Commit Protocol}

Two ensure the integrity of data proposed multilingual system follows the ACID properties of the transactions. ACID properties state that all the sites in which a transaction $T$ executed must agree on the final outcome of the execution and execution of a transaction $T$ in isolation preserves the consistency of the database. $T$ must commit at all sites or it must abort at all sites. To ensure that our system always follows ACID properties we have used two-phase commit protocol. In this proposed multilingual system, the dictionaries and encoded DB will always be consistent after the execution of any transaction at any site.

\subsubsection{Mapping between Database and Data Dictionaries}

For efficient handling of multilingual data, proper mapping between dictionaries and database is a crucial factor. Forward mapping or dictionary-to-DB mapping is needed when we want to store something in the database. Forward mapping should be handled in such a way that there will be no ambiguity in the database or dictionaries. For this purpose, care has to be taken so that appropriate code is selected and it is placed in the appropriate field of the database. On the other hand, backward mapping of DB-to-dictionary mapping is needed at the time of retrieving information. In backward mapping selection of appropriate values in appropriate language from the dictionaries based on codes should be done accurately and efficiently.

\subsubsection{Complexity Analysis}

The multilingual data management system developed has two parts for the time concern; one for searching and storing the necessary information in the dictionary and another is for dictionary and database mapping at the time of different operations. For complexity analyses of the developed multilingual system consider the following.

Size of each block so that the block entirely fits in memory $=B_{s}$

Number of blocks needed to store each of $n$ dictionaries $=D_{b}$

Number of blocks needed to store encoded table $=E_{\mathrm{b}}$

Hash function that maps each tuple of a dictionary in any of $D_{b}$ blocks $=h$

Let the multilingual operation is based on $N$ attributes that consists of $S$ text attributes and remaining numeric and alphanumeric attributes. Therefore, the complexity can be calculated as follows:

\section{Worst case:}

Worst case query complexity occurs when all the attributes involved in query are text attributes and values in the encoded table are in different blocks.

So, in such situation maximum block transfer corresponding to a query $=N+E_{b}$

Here, $E_{b}$ is the total number of block transfer of the encoded table.

\section{Best case:}

Best case query complexity occurs when all the attributes involved in query are numeric / alphanumeric attributes and values in the encoded table is in the same block.

So, in such situation maximum block transfer corresponding to a query $=1$

\section{Average case:}

Average case query complexity occurs when number of attributes involved in query are both numeric/alphanumeric and text attributes and values in the encoded table is obtained by accessing fewer blocks. So, in such cases maximum block transfer corresponding to a query that includes $S$ text attributes $=S+E_{f}$. Here, $E_{f}$ means number of block transfer corresponding to encoded table. 


\subsection{RESULTS AND DISCUSSIONS}

This section discusses the experimental setup for the simulation and the corresponding results.

\subsection{Experimental Setup}

The multilingual data management system has been developed on a machine having the operating system Windows XP, 2.4 GHz Pentium IV Processor with 512 MB memory. The system was implemented in JDK 1.6 in the front and in Oracle 9i DBMS in the back-end for storing the database, data dictionaries and related information.

For measuring the performance of the proposed multilingual system, we have considered two schemas student schema and library schema. A data generation program has generated data items of student schema. Hundred thousand records were randomly generated for different students under departments of public universities. Library schema is chosen from the central library of Chittagong University of Engineering and Technology (CUET), Bangladesh. This schema is used in CUET for maintaining books information in English. Data items of library schema are real data.

\subsection{Space Requirement Calculation}

The developed system has been implemented with six single dictionaries and one encoded table for each of the student and library schema. The dictionaries for student schema are for storing name, status, city, faculty name, department name and university name and the dictionaries for library schema are for storing book title, book author, book type, edition place, publisher and edition source. Each of these dictionaries has three fields for storing information in English, Bangla and auto incremented code for mapping dictionary to encoded DB.

In the proposed system, storage is needed for storing text data in the dictionaries. Also storage is needed for storing code corresponding to text data and numeric/alphanumeric data in the encoded table. On the other hand storage of existing systems includes the storage of information separately in each language.

Table 9: Comparative storage requirement of the developed systems and existing systems

\begin{tabular}{|c|c|c|c|c|}
\hline \multirow{2}{*}{$\begin{array}{c}\text { No. of } \\
\text { records to } \\
\text { store }\end{array}$} & $\begin{array}{c}\text { Storage } \\
\text { requirement in } \\
\text { our system in } \\
\text { MB }\end{array}$ & $\begin{array}{c}\text { Storage } \\
\text { requirement in } \\
\text { existing } \\
\text { systems in MB }\end{array}$ & $\begin{array}{c}\text { Storage } \\
\text { requirement in } \\
\text { our system in } \\
\text { MB }\end{array}$ & $\begin{array}{c}\text { Storage } \\
\text { requirement in } \\
\text { existing } \\
\text { systems in MB }\end{array}$ \\
\hline 1000 & 0.484 & 0.801 & 0.689 & 1.0872 \\
\hline 5000 & 1.989 & 4.005 & 3.086 & 5.436 \\
\hline 10000 & 3.01 & 8.01 & 3.563 & 10.872 \\
\hline 20000 & 4.721 & 16.022 & 4.516 & 21.744 \\
\hline 40000 & 7.813 & 32.042 & 6.424 & 43.488 \\
\hline 60000 & 10.901 & 48.065 & 8.331 & 65.232 \\
\hline 80000 & 13.991 & 64.087 & 10.238 & 86.976 \\
\hline 100000 & 15.631 & 81.108 & 12.146 & 108.72 \\
\hline 200000 & 23.833 & 160.218 & 21.682 & 217.44 \\
\hline
\end{tabular}

The storage requirement for different number of records shown in Table 9 for existing systems is obtained by summing up the storage required in English and Bangla. Using the data of Table 9, we have obtained the graph as shown in Fig. 3. The graph in Fig. 3 shows the comparative storage requirement for student schema and library schema. 


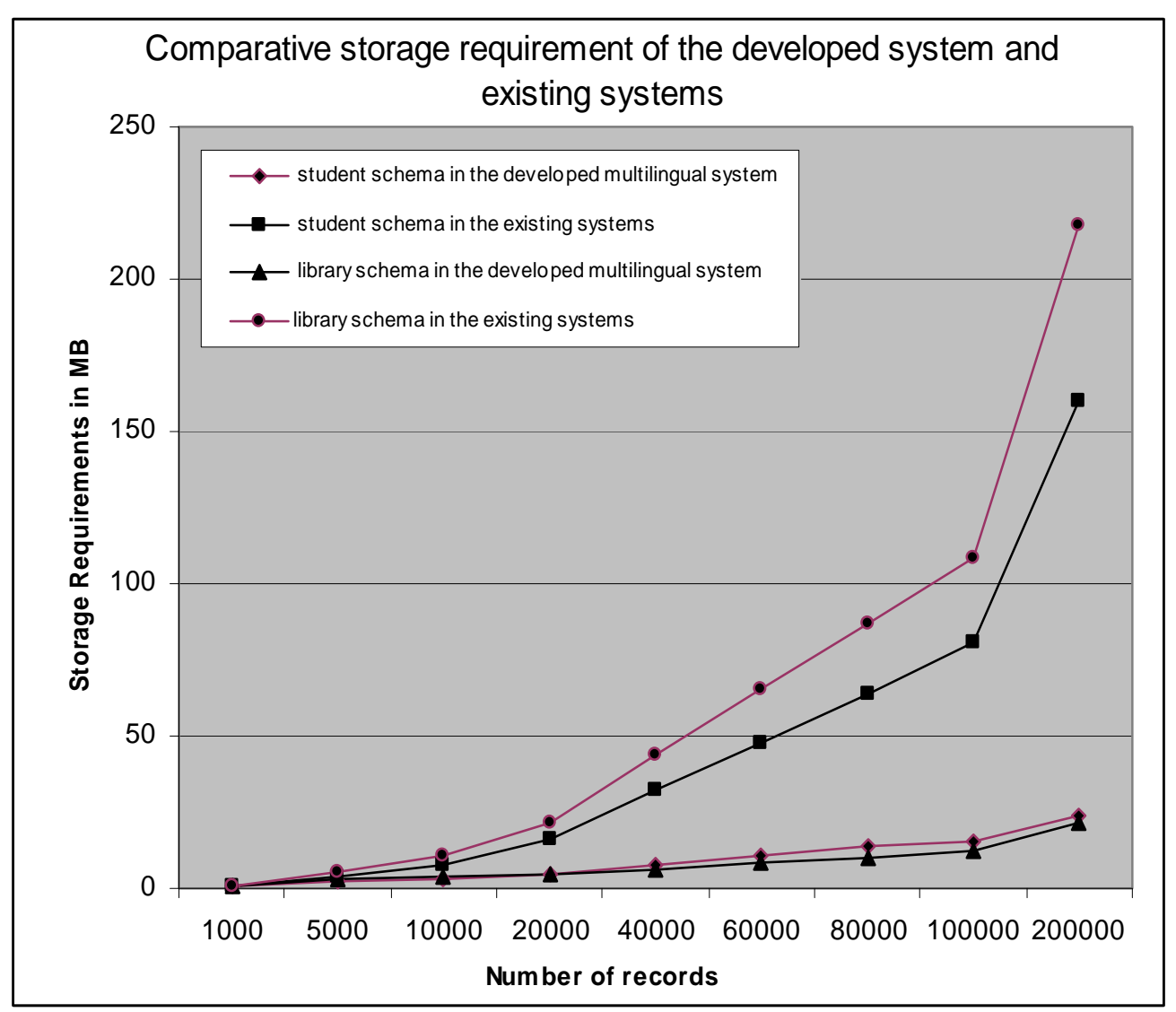

Fig. 3: Comparative storage requirement of the developed multilingual system

From Fig 3, it is observed that the rate of increase of storage requirement with the increase of number of records is significantly higher in existing systems compared to our system. This is because in the our multilingual system when dictionaries are converged there is no further entry in the dictionary and the storage of the compressed database is independent of the number of language support. But in existing systems it is necessary to insert records separately in each language.

\subsection{Multilingual Translation Performance}

For measuring the efficiency of the translator, we have applied the developed translator on different numeric, alphanumeric and text data of student schema and library schema. We have randomly chosen one thousand records of both student schema and library schema and found the translation accuracy of the proposed multilingual system. We have found the accuracy of the translator as shown in Table 10.

From the information of Table 10, we have found that the developed translator performs accurate translation of numeric and alphanumeric data and sufficient translation of text data. Also it is observed that translation of text items in case of real data set is better than random generated data set. 
Table 10: Translation accuracy of the developed translator

\begin{tabular}{|c|c|c|c|c|}
\hline \multirow{2}{*}{$\begin{array}{c}\text { No. of } \\
\text { records }\end{array}$} & $\begin{array}{c}\text { Student schema } \\
\text { translation } \\
\text { accuracy } \\
(\%)\end{array}$ & $\begin{array}{c}\text { Numeric / } \\
\text { alphanumeric } \\
\text { translation } \\
\text { accuracy (\%) }\end{array}$ & $\begin{array}{c}\text { Text translation } \\
\text { accuracy (\%) }\end{array}$ & $\begin{array}{c}\text { Numeric / } \\
\text { alphanumeric } \\
\text { translation } \\
\text { accuracy (\%) }\end{array}$ \\
\hline 100 & 76 & 100 & 73 & 100 \\
\hline 200 & 71 & 100 & 76 & 100 \\
\hline 300 & 73 & 100 & 75 & 100 \\
\hline 400 & 68 & 100 & 73 & 100 \\
\hline 500 & 77 & 100 & 78 & 100 \\
\hline 600 & 74 & 100 & 83 & 100 \\
\hline 700 & 71 & 100 & 76 & 100 \\
\hline 800 & 75 & 100 & 80 & 100 \\
\hline 900 & 72 & 100 & 82 & 100 \\
\hline 1000 & 76 & 100 & & 83 \\
\hline
\end{tabular}

This is because in real data set all the entries are valid and understandable. From our experiment we have obtained the result corresponding to translation time as shown in Fig. 4.

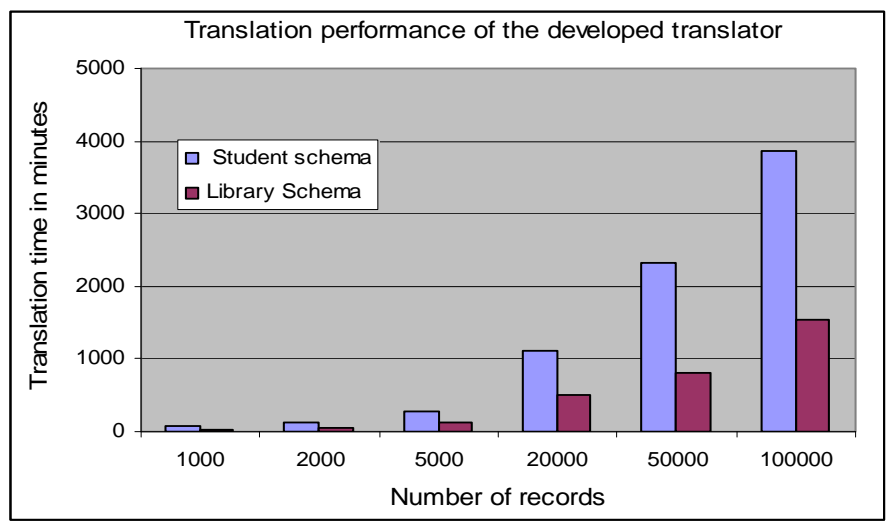

Fig. 4: Translation time requirement of the developed translator

\subsection{Multilingual Query Performance}

Query performance in our MDMS has been measured by combining the dictionary search time and database search time. For getting accurate performance, we have generated same query on different size of the database and different queries on same dataset. In our experiment, we have considered ten different criterions for performing query operation, which are given below:

- Q1: Query based on text fields and returned values are also text fields.

- Q2: Query based on numeric/alphanumeric fields and returned values are also numeric / alphanumeric fields.

- Q3: Query based on text fields and returned values are numeric/alphanumeric fields.

- Q4: Query based on numeric/alphanumeric fields and returned values are text fields.

- Q5: Query based on both text and numeric/alphanumeric fields and returned values are text fields. 
- Q6: Query based on both text and numeric/alphanumeric fields and returned values are numeric/alphanumeric fields.

- Q7: Query based on text fields and returned values are text and numeric/alphanumeric fields.

- Q8: Query based on numeric/alphanumeric fields and returned values are text and numeric/alphanumeric fields.

- Q9: Query based on both text and numeric/alphanumeric fields and returned values are text and numeric/alphanumeric fields with less text attributes than numeric/alphanumeric attributes involved in query.

- Q10: Query based on both text and numeric/alphanumeric fields and returned values are text and numeric/alphanumeric fields with more text attributes than numeric/alphanumeric attributes involved in query.

We have considered different text and numeric/alphanumeric fields for the experiment in both student schema and library schema. Using the above query conditions in both English and Bangla on different database size and averaging the result obtained in each language, we have obtained the query time for both student schema and library schema as shown in Table 11 and Table 12

Table 11: Query time in milliseconds on student schema

\begin{tabular}{|c|c|c|c|c|c|c|c|c|c|c|c|}
\hline $\begin{array}{c}\text { No. of } \\
\text { records }\end{array}$ & $\mathbf{Q 1}$ & $\mathbf{Q 2}$ & $\mathbf{Q 3}$ & $\mathbf{Q 4}$ & $\mathbf{Q 5}$ & $\mathbf{Q 6}$ & $\mathbf{Q 7}$ & $\mathbf{Q 8}$ & $\mathbf{Q 9}$ & $\mathbf{Q 1 0}$ & $\begin{array}{c}\text { Average } \\
\text { query } \\
\text { time }\end{array}$ \\
\hline 1000 & 2458 & 230 & 1163 & 1418 & 2775 & 1066.5 & 2246.5 & 1320.5 & 1780.5 & 2358 & 1682 \\
\hline 5000 & 3914 & 273 & 2529 & 2952.5 & 4326.5 & 2265 & 3523.5 & 2393.5 & 3768.5 & 4132 & 3008 \\
\hline 10000 & 4558.5 & 375 & 3515 & 3974 & 5278 & 2854.5 & 4804.5 & 3169.5 & 4314.5 & 4643.5 & 3749 \\
\hline 20000 & 6661.5 & 723.5 & 4792 & 5182.5 & 6952 & 3671 & 5786.5 & 4326.5 & 6157.5 & 6541.5 & 5079 \\
\hline 40000 & 8486.5 & 1159 & 5603 & 5935.5 & 8731 & 4495 & 6582 & 4913 & 7245.5 & 7877 & 6103 \\
\hline 60000 & 9397 & 1420 & 6603 & 7025.5 & 9287.5 & 5304.5 & 7952.5 & 5652.5 & 8378 & 8742 & 6976 \\
\hline 80000 & 10825.5 & 1601 & 7369 & 7979 & 12152.5 & 6044.5 & 8822 & 6446.5 & 9980 & 10427.5 & 8165 \\
\hline 100000 & 12200.5 & 1777 & 7974.5 & 8440 & 13707.5 & 6899 & 9734.5 & 7502 & 10841 & 11334 & 9041 \\
\hline
\end{tabular}

Table 12: Average query time in milliseconds on library schema

\begin{tabular}{|c|c|c|c|c|c|c|c|c|c|c|c|}
\hline $\begin{array}{c}\text { No. of } \\
\text { records }\end{array}$ & Q1 & Q2 & Q3 & Q4 & Q5 & Q6 & Q7 & Q8 & Q9 & Q10 & $\begin{array}{c}\text { Average } \\
\text { query } \\
\text { time }\end{array}$ \\
\hline 1000 & 2287 & 231 & 1100 & 1383.5 & 2686 & 940.5 & 2177.5 & 1279 & 1704.5 & 2265 & 1605 \\
\hline 5000 & 3788 & 278 & 2483 & 2880.5 & 4317.5 & 2255.5 & 3530 & 2343 & 3706 & 4089.5 & 2967 \\
\hline 10000 & 4529 & 392.5 & 3569.5 & 4000 & 5205 & 2737.5 & 4737 & 3172.5 & 4289 & 4246 & 3688 \\
\hline 20000 & 6631 & 705.5 & 4871.5 & 5093.5 & 6862 & 3666 & 5776 & 4232 & 6050.5 & 6516.5 & 5040 \\
\hline 40000 & 8369.5 & 1160 & 5671.5 & 6017 & 8794 & 4451.5 & 6603.5 & 4890 & 7325 & 7969.5 & 6125 \\
\hline 60000 & 9449 & 1410 & 6660.5 & 7124 & 9276.5 & 5382 & 7864 & 5704.5 & 8358.5 & 8715 & 6994 \\
\hline
\end{tabular}




\begin{tabular}{|c|c|c|c|c|c|c|c|c|c|c|c|}
\hline 80000 & 10704.5 & 1491.5 & 7420.5 & 8077 & 12375 & 6185 & 8889.5 & 6366.5 & 10088 & 10266 & 8186 \\
\hline 100000 & 12317.5 & 1841.5 & 8172 & 8321 & 13669.5 & 6852 & 9525 & 7469 & 10660 & 10741.5 & 8957 \\
\hline
\end{tabular}

For comparative performance analysis of multilingual query of the developed MDMS, we have first calculated the average query time as shown in Table 11 and Table 12 and compared this time with the time obtained using same queries (used earlier in the developed MDMS) separately in English and Bangla database on same record size (as used in the developed MDMS) and then averaging the query result obtained separately in English and Bangla. From the experiment, we have obtained the comparative query performance of the developed MDMS and existing systems as shown in Fig. 5.

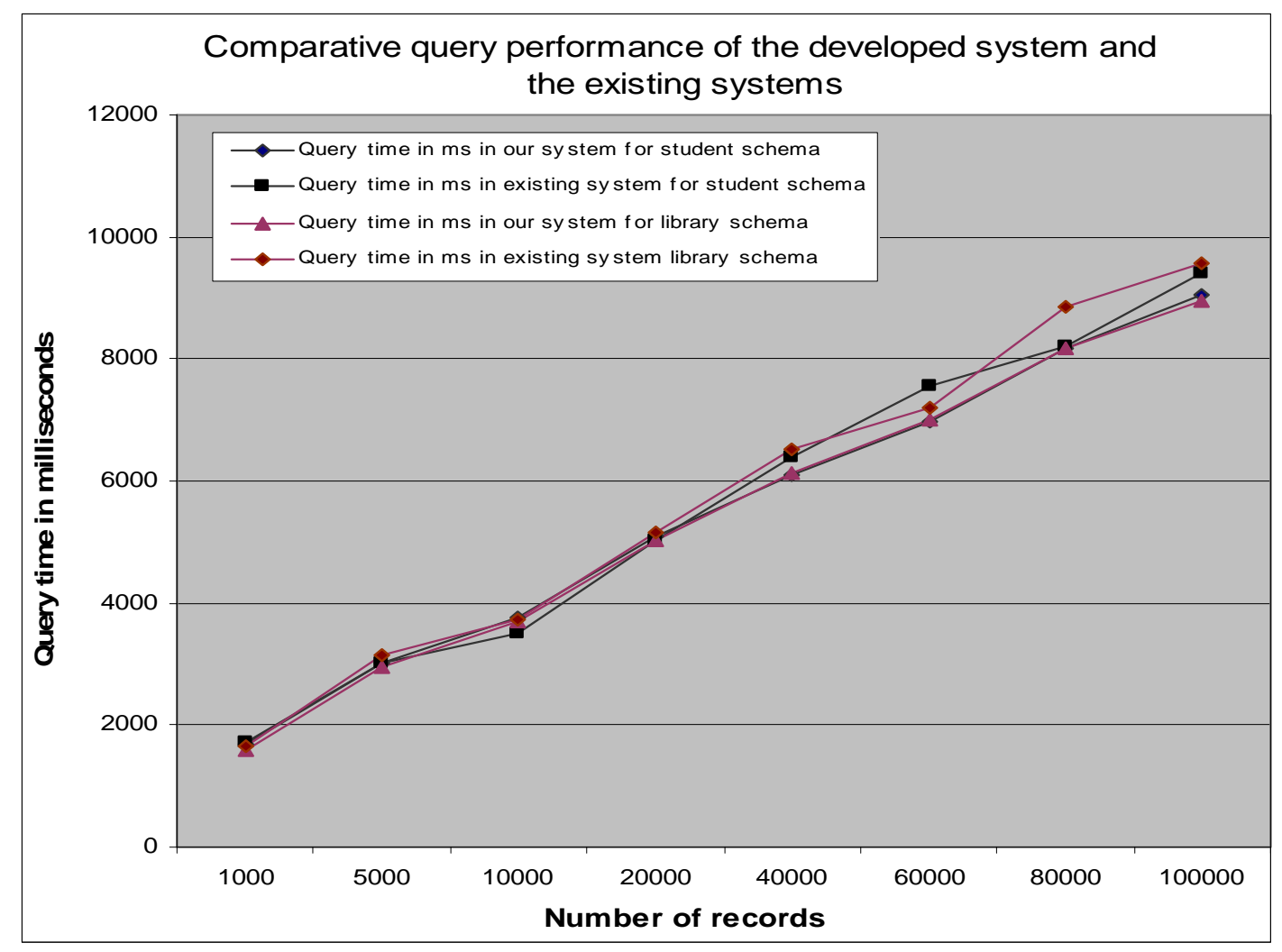

Fig. 5: Comparative multilingual query performance

From Fig. 5, it is observed that multilingual query performance of the developed system is slightly better than the query time of the existing systems. This is because in the developed multilingual system, we need to perform query operation in a single encoded database and the query operation is independent of language selection. But in existing systems we have to use redundant record sets and query operation need to be performed separately from language dependent databases separately to retrieve same data in different languages.

\subsection{Multilingual Insertion Performance}

For the experimental purpose of insertion, we have considered the matter of random record generation. In this approach when we insert a record it will insert eight text fields, which includes name, father's name, mother's name, city, marital status, department, faculty and university and seven numeric and alphanumeric fields, which includes student id, course id, 
level, term, average marks obtained in a specific term and merit position. From our experiment we have obtained the graph shown in Fig. 6.

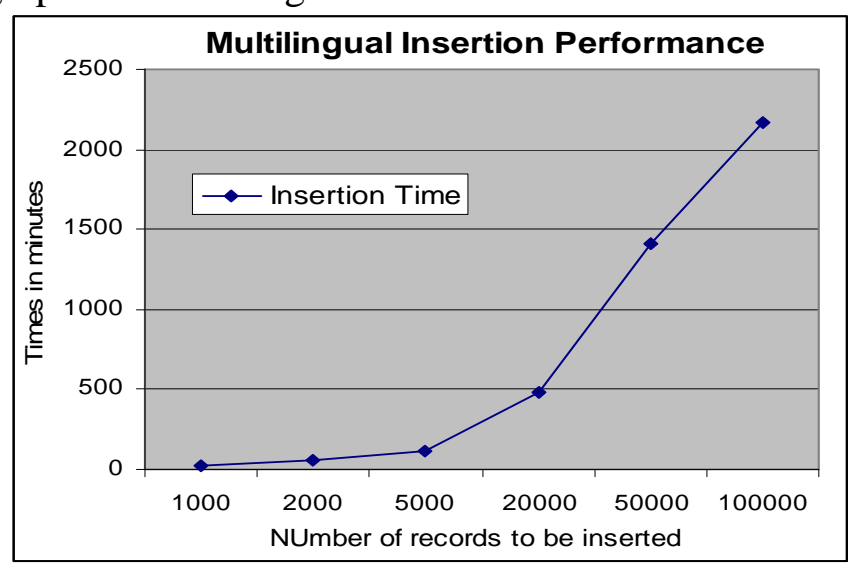

Fig. 6: Multilingual insertion performance

From the graph shown in Fig. 6, it can be considered that if the number of text fields to be inserted increases the insertion time also increases.

\subsection{Multilingual Deletion Performance}

For the deletion we have to search the record first. In the experiment we have search the records to be deleted using numeric/alphanumeric attributes, as searching using numeric/alphanumeric attributes require less time. From the experiment, we have found the information as shown in Fig.7 while performing multilingual deletion operation.

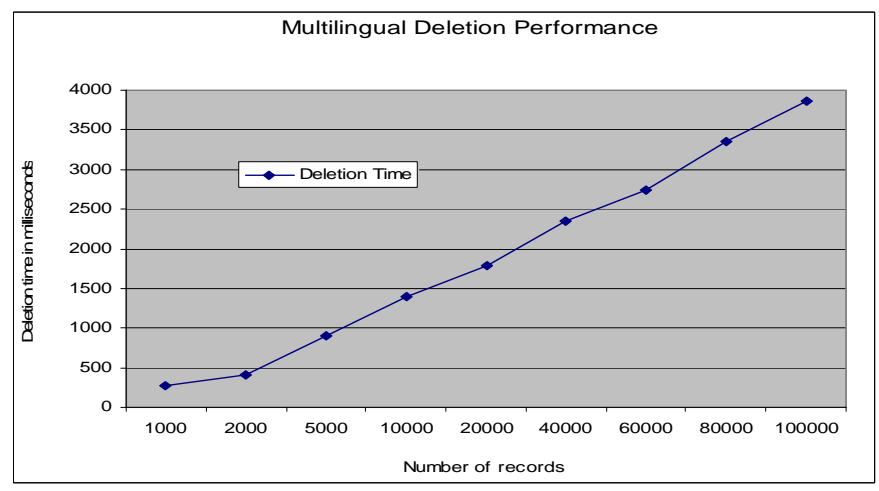

Fig. 7: Multilingual deletion performance

From the Fig. 7, it has been observed that deletion is not so much time consuming with the variation of records. This is because we performed search for tuples to be deleted based on numeric/alphanumeric attributes and deleted tuples directly from the database without involvement of dictionaries.

\subsection{Multilingual Update Performance}

Update performance is measured by summing up the time required to perform query operation to retrieve the data to be updated and the time required to insert the new data. In our developed MDMS, we have retrieved data to be updated using query based on studentid for student schema and subjectcode for library schema. For measuring update performance in the developed 
MDMS, we have generated same update operation on different size of the data set in both English and Bangla.

In our experiment, we have considered five different conditions for performing update operation, which are given below.

- U1: Updated values are text data already in the dictionary.

- U2: Updated values are text data not in the dictionary.

- U3: Updated values are numeric/alphanumeric values.

- U4: Updated values involve text data and numeric/alphanumeric data with text data in the dictionary.

- U5: Updated values involve text data and numeric/alphanumeric data with text data not in the dictionary.

We have also considered different fields to be updated. Considering conditions and number of fields to be updated, we have obtained the result as shown in Table 13.

Table 13: Update time in milliseconds on different number of records in student schema and library schema

\begin{tabular}{|c|c|c|c|c|c|c|c|c|c|c|c|c|}
\hline \multirow{2}{*}{$\begin{array}{c}\text { No. } \\
\text { of } \\
\text { Records }\end{array}$} & $\mathbf{6 1}$ & $\mathbf{U 2}$ & $\mathbf{U 3}$ & $\mathbf{U 4}$ & $\mathbf{U} \mathbf{4}$ & $\begin{array}{c}\text { Average } \\
\text { update } \\
\text { time }\end{array}$ & $\mathbf{U 1}$ & $\mathbf{U 2}$ & $\mathbf{U 3}$ & $\begin{array}{c}\text { U4 } \\
\text { U5 }\end{array}$ & $\begin{array}{c}\text { Average } \\
\text { update } \\
\text { time }\end{array}$ \\
\hline 1000 & 1695 & 2391 & 448 & 1439 & 2162 & 1627 & 1524 & 2278 & 403 & 1384 & 2012 & 1520 \\
\hline 5000 & 3915 & 4781 & 815 & 3050 & 4461 & 3404 & 3819 & 4562 & 709 & 2835 & 4528 & 3290 \\
\hline 10000 & 5481 & 6335 & 1068 & 4637 & 5987 & 4701 & 5423 & 6412 & 958 & 4682 & 5944 & 4683 \\
\hline 20000 & 7126 & 8036 & 1197 & 6138 & 7824 & 6064 & 6981 & 7836 & 1120 & 6045 & 7819 & 5960 \\
\hline 40000 & 8235 & 9148 & 1352 & 7935 & 9058 & 7145 & 8151 & 9125 & 1273 & 7889 & 9025 & 7092 \\
\hline 60000 & 9227 & 11520 & 1473 & 8944 & 10869 & 8406 & 9113 & 10521 & 1365 & 8812 & 10726 & 8107 \\
\hline 80000 & 11243 & 12782 & 2268 & 10543 & 12065 & 9780 & 10983 & 12659 & 2178 & 10223 & 11954 & 9599 \\
\hline 100000 & 12198 & 14936 & 2504 & 11087 & 14684 & 11081 & 11025 & 14561 & 2518 & 11034 & 14869 & 10801 \\
\hline
\end{tabular}

From Table 13, it is observed that in the developed MDMS maximum update time required when updated values is not in the dictionaries and minimum update time required when update is performed on numeric or alphanumeric data. This is because if updated values are not in the dictionaries, we have to insert the items in the dictionaries first and then we have to make replacement in the encoded DB with the new generated codes. On the other hand if the updated values are numeric or alphanumeric data, we can update the values directly from the encoded DB without the involvement of dictionaries.

For comparative update performance analysis we have done the following:

- Calculated the average update time considering different update conditions on same number of records (as shown in Table 13).

- Calculated the average update time on different size of records considering different update conditions. 
- Compared the time obtained in the developed MDMS with the time obtained by performing same update operation separately in English and Bangla database on the same record size and the summing up the update result obtained in English and Bangla.

From our experiment we have obtained comparative update performance as shown in Fig. 8.

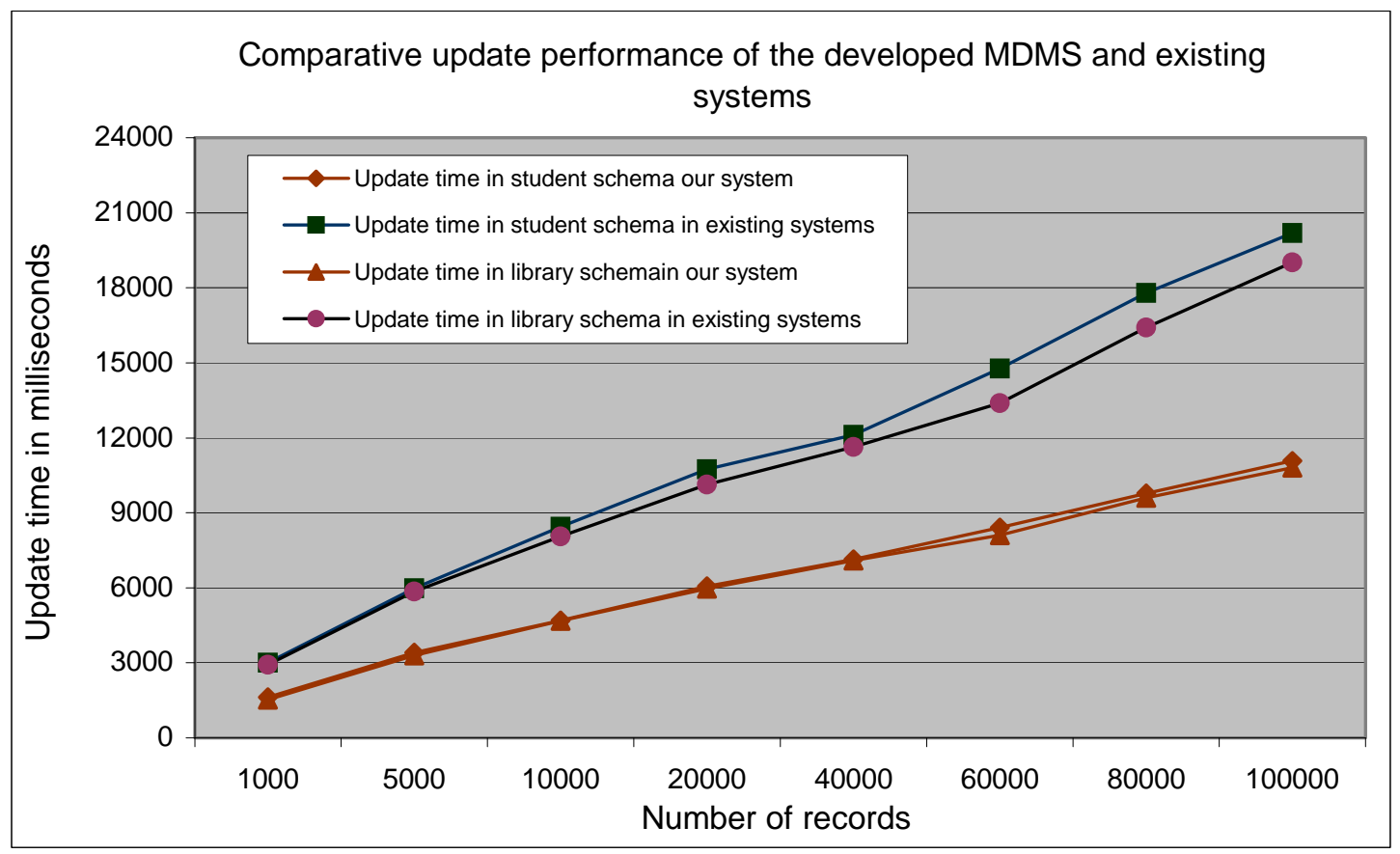

Fig. 8: Comparative update performance of the developed system

From Fig. 8, it has been observed that the developed MDMS required less update time for any update operation compared with the update operations performed in the existing systems. This is because in our system we need to perform update operation on a single encoded database. But in existing systems we have to use redundant databases and update operation need to be performed in each of the databases separately for keeping consistency of information stored in different languages.

\section{CONCLUSION}

Storage of multilingual data and support of dynamic update is a problem for conventional Database Management Systems. While existing database systems provide some means of storing and querying multilingual data, they suffer from redundancy proportional to the number of language support. In this paper, we have proposed a translator-based approach for handling multilingual data that stores data in information theoretic way with minimum redundancy. We have developed algorithms for insertion of multilingual data into a single non-redundant database, querying and update in the database. The algorithms have been evaluated by syntactic data sets generated by a data generation program and real data sets as well. We have compared the performance of our system with the existing systems. Our system outperforms the existing multilingual systems in terms of both space and time.

We have implemented the system for two languages: English and Bangla. In this system, data has been stored in a central server and clients can perform different operations in the database dynamically in a distributed environment. Schema evolution in the developed MDMS is simple 
and easier to maintain the database consistency because of a single encoded database. These tasks are difficult in the existing systems due to redundant databases. Query performance in the proposed system is more efficient than the existing systems. Though we have considered Bangla and English languages for the experimental purpose, the system can adapt other languages with the addition of the corresponding language translator only.

\section{REFERENCES}

[1] C. Ordonez, "Optimizing Recursive Queries in SQL", Proceedings of the ACM SIGMOD International Conference on Management of Data, pp 834-839, 2005.

[2] W. Chung, Y. Zhang, Z. Huang, G. Wang, T. H. Ong and T. H. Chen, "Internet Searching and Browsing in a Multilingual World: An Experiment on the Chinese Business Intelligence Portal ", Journal of the American Society for Information Science and Technology, Vol. 55, No. 9, pp 818-831, 2004.

[3] A. Kumaran, P. Chowdary and J. Haritsa "On pushing multilingual query operators into relational engines”, Proceedings of 22nd IEEE International Conference on Data Engineering (ICDE), Atlanta, USA, April 2006.

[4] F. Gey, A. Chen, M. Buckland and R. Larson, "Translingual vocabulary mapping for multilingual information access", Proceedings of the 25th Annual International ACM SIGIR Conference on Research and Development in Information Retrieval, Tampere, Finland, pp 455-456, August 2002.

[5] J. Marlow, P. Clough, J. C. Recuero and J. Artiles, "Exploring the Effects of Language Skills on Multilingual Web Search", In Proceedings of the 30th European Conference on IR Research (ECIR'08), Glasgow, UK, April 2008.

[6] The Computer Scope Ltd., Dublin, Ireland. http://www.NUA.ie/Surveys

[7] A. Kumaran and J. R. Haritsa, "On the Costs of Multilingualism in Database Systems", Proceedings of 29th Very Large Databases Conference, Berlin, Germany, pp 105-116, September 2003.

[8] A. Kumaran,.. "Multilingual information processing on relational database architectures", Ph.D Thesis, Department of Computer Science and Automation, Indian Institute of Science, Bangalore, India, December 2005.

[9] A. Silberschatz, H. F. Korth, and S. Sudarshan, "Database systems concepts", Fourth Edition, Tata McGraw Hill, pp 709 - 730, 2002.

[10] The Association for Computational Linguistics. http://www.aclweb.org

[11] Special Interest Group in Information Retrieval (ACM SIGIR). http://www.acm.org/sigir.

[12] P. Pingali and V. Varma, "Multi-lingual Indexing Support for CLIR using Language Modeling”, Bulletin of the Technical Committee on Data Engineering, IEEE Computer Society, Vol. 30, No. 1, pp 57-72, March 2007

[13] R. King and A. Morfeq, "Bayan: An Arabic Text Database Management System", Proceedings of the 1990 ACM SIGMOD International Conference on Management of Data, Vol. 19, Issue. 2, pp 12-23, 1990.

[14] C. Lu, and K. Lee, "A Multilingual Database Management System for Ideographic Languages”, Chinese University of Hong Kong Technical Report, 1992.

[15] M. Andersson, Y. Dupont, S. Spaccapietra, K. Yetongnon, M. Tresch and H. Ye, "FEMUS: A Federated Multilingual Database System”, Advanced Database Systems, Springer-Verlag, Vol. 359, pp 359-380, 1993.

[16] C. Yip, B. Kao and D. Cheung, "A Framework for the Support of Multilingual Computing Environments”, Technical Report, University of Hong Kong, 1997. 
[17] B. Lambert, K. Chang and S. Lin, "Descriptive Analysis of the Drug Name Lexicon" Drug Information Journal, Vol. 35 No. 1, 2001.

[18] The EROS System.http://merovingio.c2rmf.cnrs.fr/eros/eros.xhtml

[19] IBM Corporation, Armonk, New York. http://www.ibm.com

[20] Microsoft Corporation, Redmond, Washington. http://www.microsoft.com.

[21] Oracle Corporation, Redwood Shores, California. http://www.oracle.com.

\section{BIOGRAPHY}

Dr. Abu Sayed Md. Latiful Hoque received his $\mathrm{PhD}$ in the field of Computer \& Information Science from University of Strathclyde, Glasgow, UK in 2003 with Commonwealth Academic Staff Award. He obtained M.Sc. in Computer Science \& Engineering and B.Sc. in Electrical \& Electronic Engineering from Bangladesh University of Engineering \& Technology (BUET) in 1997 and 1986 respectively. He has been working as a faculty member in the Department of Computer Science \& Engineering of BUET since 1990 and currently his position is an Associate Professor. He is a Fellow of Institute of Engineers Bangladesh (IEB) and Bangladesh Computer Society. His research interest includes Data Warehouse, Data Mining, Information Retrieval and Compression in DatabaseSystems.

Mohammad Shamsul Arefin received his B.Sc. Engineering in Computer Science and Engineering from Khulna University, Khulna, Bangladesh in 2002, and completed his M.Sc. Engineering in Computer Science \& Engineering in 2008 from Bangladesh University of Engineering \& Technology (BUET), Bangladesh. He is a member of Institute of Engineers Bangladesh (IEB) and currently working as an Assistant Professor of Chittagong University of Engineering \& Technology, Chittagong, in Computer Science \& Engineering Department. His research interest includes Data Management, Semantic Web, Machine Learning, Natural Language Processing and Object Oriented System Design. 